\title{
Familial genetic tuberous sclerosis complex associated with bilateral giant renal angiomyolipoma: A case report
}

\author{
LINA WANG $^{1 *}$, DAWEI NI ${ }^{1,2 *}$, LIN ZHONG $^{3}$ and JIANBO WANG ${ }^{1}$ \\ ${ }^{1}$ Department of Urology, The First Affiliated Hospital of Dalian Medical University, Dalian, Liaoning 116011; \\ ${ }^{2}$ Department of Urology, The Second People's Hospital of Hefei, Hefei, Anhui 230011; ${ }^{3}$ Department of Pathology, \\ The First Affiliated Hospital of Dalian Medical University, Dalian, Liaoning 116011, P.R. China
}

Received December 1, 2015; Accepted June 9, 2017

DOI: 10.3892/ol.2017.7165

\begin{abstract}
Tuberous sclerosis complex (TSC) is an autosomal dominant disease involving multiple organs, but there are a limited number of reports on family TSC. In the present report, a case of a 52-year-old female with a familial genetic TSC, associated with bilateral giant renal angiomyolipoma, was described. The mother, second elder brother and daughter of the patient all exhibited TSC, but the clinical manifestations, and therapeutic prognosis between the family members were not the same. The present case report aimed at identifying an effective diagnostic method and treatment through additional study of familial genetic TSC, in order to prolong and improve the quality of life for patients with TSC. According to the present case and relevant literature reviews, it is suggested that fetal gene detection during pregnancy could prevent the passing of this disease onto further generations. Furthermore, early application of drug treatment may control the development of the disease in diagnosed patients. The combination of classical treatments with a small dose of mammalian target of rapamycin inhibitors is the typical recommendation, which may control the development of the disease more effectively and decrease adverse side-effects.
\end{abstract}

\section{Introduction}

Tuberous sclerosis complex (TSC) is an autosomal dominant disease, which typically causes benign tumors to develop in

Correspondence to: Professor Jianbo Wang, Department of Urology, The First Affiliated Hospital of Dalian Medical University, 193 Lianhe Road, Dalian, Liaoning 116011, P.R. China

E-mail: wangjianbo@dlmedu.edu.cn

Mr. Lin Zhong, Department of Pathology, The First Affiliated Hospital of Dalian Medical University, 193 Lianhe Road, Dalian, Liaoning 116011, P.R. China

E-mail: zhonglin_1983@163.com

\section{${ }^{*}$ Contributed equally}

Key words: family, tuberous sclerosis complex, angiomyolipoma, mammalian target of rapamycin, estrogen the brain and other vital organs, including the kidney, skin and liver $(1,2)$. Global statistics from previous studies, performed in 2000 and 2011, revealed that $1 / 6,000-10,000$ people are born with TSC and there are 2,000,000 people with TSC $(3,4)$ TSC is a genetic disease, with $33 \%$ of patients with TSC having inherited the disease, and $66 \%$ of patients with TSC having developed the disease due to a gene mutation (5). A definitive diagnosis of TSC often depends on clinical manifestations and genetic testing, both of which can make definitively diagnosing the patient challenging. The majority of previous studies have described individual cases, but family heredity cases are limited (6). Identifying and studying cases of familial hereditary TSC may improve early genetic diagnosis, and thus enable early intervention, which may increase the quality of life for patients with TSC. The present case report described different clinical manifestations and therapeutic prognoses of four patients with TSC from one family. Written informed consent was obtained from all patients.

\section{Case report}

A 52-year-old female, with a 20-year history of TSC, presented at the First Affiliated Hospital of Dalian Medical University (Dalian, China) with abdominal distention, abdominal pain and anorexia for the past 2 months. The patient, hereafter known as patient 1 , had epilepsy for 50 years with a mean seizure frequency of 2-3 times/month, and schizophrenia for 12 years with intelligence degeneration. The patient had been taken antiepileptic and antipsychotic drugs but the symptoms were not well controlled. Physical examination (Fig. 1) revealed facial angiofibromas beside the nasal alar (Fig. 1A), an enlarged abdomen (Fig. 1B), where a large smooth bilateral mass was observed with a nonclear boundary, and ungual fibromas (Fig. 1C and D). Biochemical tests demonstrated that the blood creatinine level was $91 \mu \mathrm{mol} / 1$, red blood cell count was $2.98 \times 10^{9}$ cells $/ 1$ and hemoglobin level was $90 \mathrm{~g} / 1$. Other lab tests, including the testing of cruor, liver function and electrolyte, revealed normal results.

Fundus examination results revealed suspicious retinal pigment spots (Fig. 1K and L). An abdominal color Doppler ultrasound indicated that there were intrahepatic calcifications and the abdominal cavity exhibited hyperechoic light mass shadows on either side, which were considered renal 
A

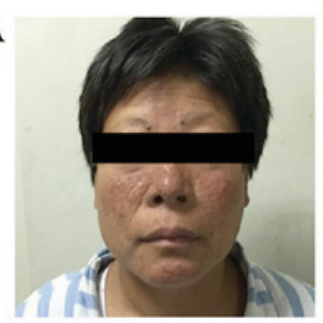

E
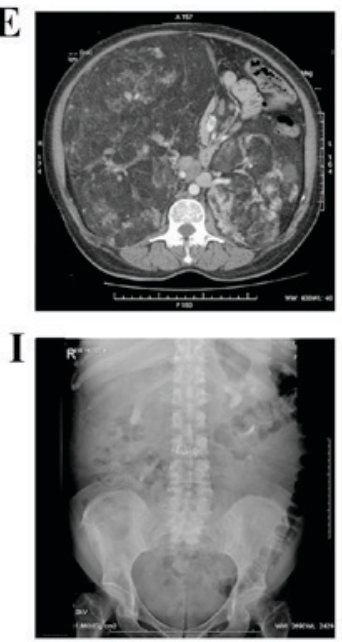

B
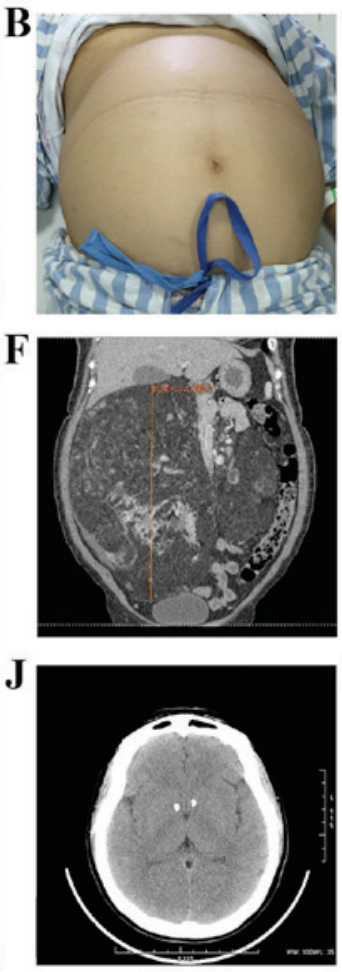
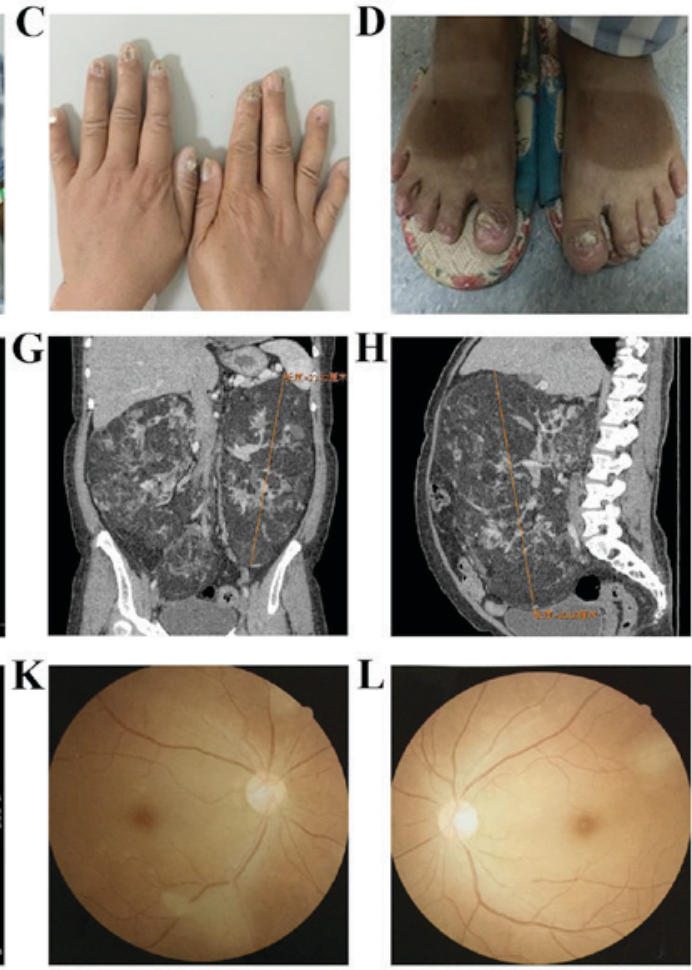

Figure 1. External characteristics and imaging performance of patient 1 with tuberous sclerosis complex. Images demonstrated (A) facial angio fibromas beside the nasal alar; (B) enlarged abdomen; (C) ungual fibromas; and (D) ungual fibromas. (E) CT scan changes in cross section. (F) Coronal section; (G) coronal section; (H) sagittal view. (E-H) Revealed CT scan changes in different sections. The masses were $28 \times 25 \times 26$ and $23 \times 15 \times 11 \mathrm{~cm}$ on the right, and left sides, respectively. The CT values were -61 and -20HU. (I) Intravenous urography revealed bilateral partial hydronephrosis. (J) Head CT identified multiple calcification. (K) Fundus examination of the left result demonstrated a suspicious retinal pigment spot. (L) Fundus examination of the right result revealed a suspicious retinal pigment spot. CT, computed tomography.

angiomyolipoma (RAML). Abdominal computed tomography (CT) (Fig. 1E-H) revealed that large bilateral mass shadows replaced the normal renal morphology. The masses were determined to be $28 \times 25 \times 26$ and $23 \times 15 \times 11 \mathrm{~cm}$ on the right, and left sides, respectively. The CT values were between-61 and -20HU. Intravenous urography (IVU; Fig. 1I) demonstrated bilateral partial hydronephrosis and multiple calcification lesions were identified in the head CT (Fig. 1J). The present case was diagnosed as TSC, according to its clinical diagnostic criteria (7). Treatment advice was administered to the patient where surgery was recommended and preoperative alternative arterial embolization could be chosen to decrease the intraoperative bleeding risk. Renal transplantation or long-term replacement therapy of hemodialysis could be applied following surgery. Mammalian target of rapamycin (mTOR) inhibitors, including sirolimus and everolimus, were selected as suitable treatment options. Furthermore, radiofrequency ablation with a small dose of mTOR inhibitors was suggested. However, due to the high risk of the surgery and the high drug costs, the patient refused surgical, and drug treatments.

Tracing the disease in the family of the patients revealed that four family members experienced the disease successively, which included the patient (patient 1), the patient's mother (patient 2), the patient's second elder brother (patient 3) and the patient's daughter (patient 4; Fig. 2). However, the clinical manifestations were not exactly the same (Table I). Patient 2 exhibited facial angiofibromas, ungula fibromas and intelligence degeneration. In addition, bilateral RAML was observed and two surgeries were performed (left nephrectomy

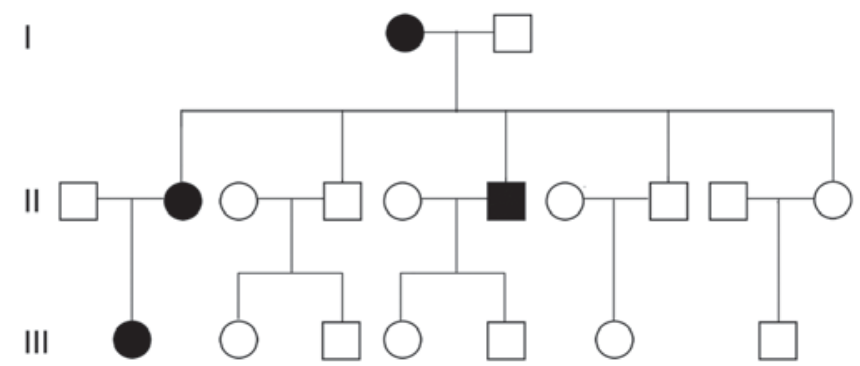

Figure 2. Genetic map illustrating the inheritance of tuberous sclerosis complex in one family. Filled circles and squares represent affected females and males, respectively. I, II and III represent the first, second and third generations of the family, respectively. Patient 1 , filled circle in the second generation; patient 2, filled circle in the first generation; patient 3 , filled square in the second generation; and patient 4 , filled circle in the third generation.

and right nephron-sparing surgery); however, the patient's mother succumbed to other diseases.

Patient 3, 55 years of age at present, was admitted to the First Affiliated Hospital of Dalian Medical University (Dalian, China) in June 2002, presenting with a 3-month history of bilateral waist discomfort. Patient 3 had epilepsy for $>12$ years without intelligence degeneration, and did not take medicine regularly. Physical examination revealed facial angiofibromas and ungual fibromas. An abdominal CT identified a soft tissue mass in the left kidney (size, 6x7 cm) and multiple uniform density masses in the right kidney (size, between 0.5 and $2 \mathrm{~cm}$ ). Furthermore, a head CT demonstrated 
Table I. Common and distinct clinical manifestations observed in four patients, from the same family, with tuberous sclerosis complex.

\begin{tabular}{|c|c|c|c|c|}
\hline $\begin{array}{l}\text { Clinical manifestation and } \\
\text { imaging examination }\end{array}$ & Patient 1 & Patient 2 & Patient 3 & Patient 4 \\
\hline Facial angiofibromas & + & + & + & + \\
\hline Ungual fibromas & + & + & + & Fingernail - toenails + \\
\hline Retinal pigment spots & $+/-$ & - & - & - \\
\hline Shagreen patch & - & - & - & + \\
\hline Intelligent degeneration & + & + & - & + \\
\hline Seizure history & + & - & + & - \\
\hline Head CT & Multiple calcification & Not done & Multiple calcification & $\begin{array}{c}\text { Subependymal } \\
\text { giant cell astrocytoma } \\
\text { and calcification }\end{array}$ \\
\hline Bilateral renal AML & + & + & + & + \\
\hline Liver CT & Calcification & - & - & Angiofibromas \\
\hline
\end{tabular}

CT, computed tomography; AML, angiomyolipoma; +, positive; -, negative.
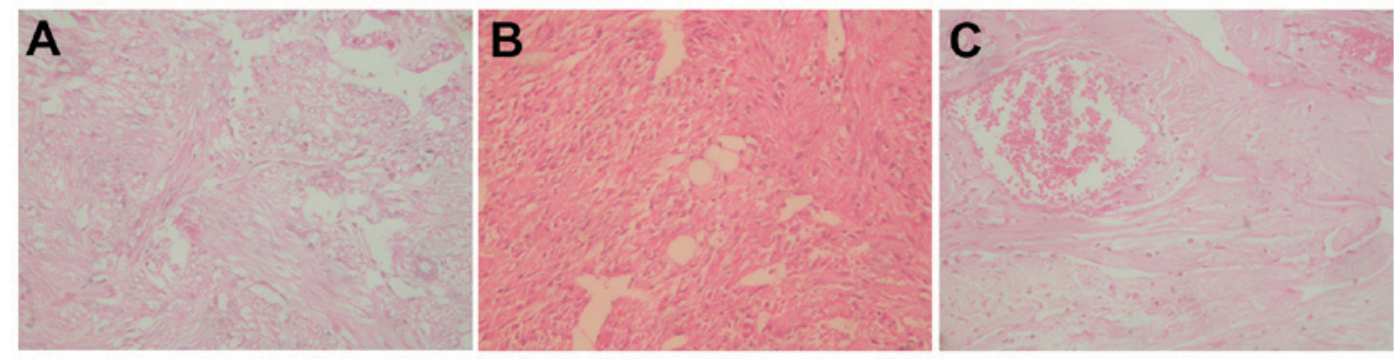

Figure 3. Hematoxylin and eosin stain of patient 3 indicates classical angiomyolipoma: (A) Smooth muscle cell; (B) fat composition is absent; (C) blood vessel (magnification, $\mathrm{x} 40$ ).

calcification beside the two cerebral ventricles. Biochemistry test results were all normal, with the exception of blood creatinine, which was $84 \mu \mathrm{mol} / 1$. According to the examination results, a left nephrectomy was performed. The surgery and recovery were successful; however, image documents were lost and patient 3 moved to a different city. The patient was followed up via the telephone and the general condition of the patient improved. Fig. 3 presents a hematoxylin and eosin stain, which revealed a classical RAML structure with a majority of smooth muscle and an absence of fat composition. The procedure for hematoxylin and eosin staining included dewaxing, with xylene, staining (hematoxylin, 2 min; and eosin, $3 \mathrm{~min}$; both at room temperature), dehydrating, transparentizing and mounting. Sections were $5 \mu \mathrm{m}$. The procedure for immunohistochemistry included dewaxing, hydration, antigen retrieval, primary antibody incubation, secondary antibody incubation, streptaridin-peroxidase reaction, color development, redyeing, dehydrating, transparentizing and mounting. Tissues were paraffin embedded, the fixative used was $10 \%$ formalin, at room temperature for between 30 and $50 \mathrm{~min}$. Sections were placed in an oven at $60^{\circ} \mathrm{C}$ for $20 \mathrm{~min}$ and subsequently in xylene solution for $10 \mathrm{~min}$ two times. Sections were placed in $100 \%$ absolute ethanol for $5 \mathrm{~min}$ two times, $95 \%$ ethanol for $2 \mathrm{~min}, 80 \%$ ethanol for $2 \mathrm{~min}$, $70 \%$ ethanol for $2 \mathrm{~min}$, distilled water for $5 \mathrm{~min}$, and washed with PBS for 5 mins three times. Following this, sections were blocked with 5\% normal goat serum (Shanghai Jianglai Biotech Co., Ltd., Shanghai, China) at room temperature for $20 \mathrm{~min}$, incubated with $3 \% \mathrm{H}_{2} \mathrm{O}_{2}$ for $10 \mathrm{~min}$ at room temperature, to block the activity of endogenous peroxidase, and washed with PBS (three times, 5 min each). Sections were subsequently incubated with primary antibodies overnight at $4^{\circ} \mathrm{C}$. Primary antibody details are listed in Table II. Following this, the MaxvisionTM Kit (rabbit) was used (cat. no. KIT-5030; Fuzhou Maixin Biotech Co., Ltd., Fuzhou, China) for secondary antibody incubation $\left(37^{\circ} \mathrm{C}\right.$ for $\left.30 \mathrm{~min}\right)$. Subsequently, sections were washed with PBS five times for 5 min each. Chromogen was detected using 3,3'-diaminobenzidine and sections were stained with haematoxylin at room temperature for $5 \mathrm{sec}$, for nuclear protein, and $50 \mathrm{sec}$, for endochylema (or envelope protein). A light microscope was used at magnification, x100.

Immunohistochemistry results identified the following: Homatropinemethylbromide45-positive; 5- $(\mathrm{N}$, $\mathrm{N}$-Hexamethylene) amiloride (HMA)-positive; actin-positive; desmin-positive; vimentin-positive; cluster of differentiation (CD)34-positive; CD68-sparsely positive; S-100 protein-negative; CD10-negative; epithelial membrane antigen-negative; cytokeratin-negative; Ki-67 protein $<1 \%$-positive (Fig. 4). The pathological diagnosis was classical RAML. 
Table II. Primary antibody details.

\begin{tabular}{ll}
\hline Antibody & \multicolumn{1}{c}{ Cat. no. } \\
\hline Melanoma & MAB-0098 \\
$\beta$-actin & Kit-0032 \\
CD34 & Kit-0004 \\
CD68 & MAB-0041 \\
Desmin & Kit-0023 \\
Actin (smooth muscle) & Kit-0006 \\
Ki-67 antigen & Kit-0005 \\
Vimentin & Kit-0019 \\
CD10/calla & MAB-0338 \\
Pan-cytokeratin & Kit-0009 \\
EMA & Kit-0011 \\
S-100 Protein & Kit-0007
\end{tabular}

All antibodies were purchased from Fuzhou Maixin Biotech Co., Ltd. $\mathrm{CD}$, cluster of differentiation; EMA, epithelial membrane antigen.

Patient 4, 24 years of age at present, was diagnosed with TSC in November 2009. At the latest return visit (June 2015), intelligence decreased without record of epilepsy. Patient 4 exhibited facial angiofibromas; however, patient 4 also exhibited a shagreen patch on the back (size, $5 \times 3 \mathrm{~cm}$ ), which the other members of this family did not have. Although fibroma was developed under the toenails of patient 4 , the fingernails were observed to be normal. Biochemical tests revealed the following results, which were all in the normal range: Red blood cell count, $3.84 \times 10^{9}$ cells/l; and hemoglobin, $113 \mathrm{~g} / \mathrm{l}$. The renal CT, performed 6 years prior, revealed multiple angiomyolipoma (AML) in the two kidneys (diameter, between 0.5 and $4 \mathrm{~cm}$ ), but the renal morphology was normal. Compared with the previous $\mathrm{CT}$, at present, the number and the volume of AML increased significantly. The largest AMLs in the left and right kidneys were determined to be $15 \times 11 \times 8$ and 17x 10x9 cm, respectively. The CT value was between- 80 and 23HU (Fig. 5A and B). The bilateral renal morphology was vague and the renal parenchyma were eroded. No lesion was identified in the liver 6 years prior, but multiple hemangiomas were now observed (Fig. 5D and E). IVU (Fig. 5C) revealed that the right upper calyceal and the left inferior calyceal were not developed. Furthermore, a head CT (Fig. 5F) identified a subependymal giant cell astrocytoma in the septum pellucidum near the interventricular foramen (diameter, $1.2 \mathrm{~cm}$ ), and multiple calcification lesions were observed. A comparison between head CT scans was not conducted as patient 4 did not have a head CT performed 6 years prior. A combination of surgical with medical treatments, including everolimus and sirolimus, was suggested, and the necessity of treatments was introduced, but the patient refused therapy.

\section{Discussion}

TSC is an autosomal dominant disease, which typically develops in the kidneys, brain, heart, skin, liver and a number of other organs, and exhibits individual differences, in spite of patients exhibiting the same type of genetic mutation (8).
Table III. Clinical diagnostic criteria of tuberous sclerosis complex.
A, Major features
1. Hypomelanotic macules ( $\geq 3$; diameter, $\leq 5 \mathrm{~mm}$ )
2 . Angiofibromas $(\geq 3)$ or fibrous cephalic plaque
3. Ungual fibromas $(\geq 2)$
4. Shagreen patch
5. Multiple retinal hamartomas
6. Cortical dysplasias ${ }^{\mathrm{a}}$
7. Subependymal nodules
8. Subependymal giant cell astrocytoma
9. Cardiac rhabdomyoma
10. Lymphangioleiomyomatosis ${ }^{\mathrm{b}}$
11. Angiomyolipomas $(\geq 2)^{\mathrm{b}}$

\section{B, Minor features}

1. 'Confetti' skin lesions

2. Dental enamel pits $(>3)$

3. Intraoral fibromas $(\geq 2)$

4. Retinal achromic patch

5. Multiple renal cysts

6. Nonrenalhamartomas

Includes tubers and cerebral white matter radial migration lines. ${ }^{\mathrm{b}} \mathrm{A}$ combination of the two major clinical features (LAM and angiomyolipomas) without other features does not meet criteria for a definite. Definite diagnosis, 2 major features or 1 major feature with $\geq 2$ minor features; possible diagnosis, either 1 major feature or $\geq 2$ minor features.

It has been identified that the genes associated with TSC are TSC1 and TSC2, which are two tumor suppressor genes (9) TSC1 is located at region q34 of chromosome 9 and encodes the protein Hamartin, and TSC2 is located at region p13 of chromosome 16 and encodes the protein Tuberin $(9,10)$. The Hamartin-Tuberin protein complex may suppress the transduction of mTOR pathway (11). Once TSC1 and TSC2 are mutated, the Hamartin-Tuberin protein complex cannot form, thus activating the mTOR signaling pathway and resulting in benign tumors involving a number of organs all over the body (12). The lesions typically occur in the brain, kidney, heart, liver, pulmonary and skin, causing epilepsy, mental retardation, autism, dysfunctions of liver, lung, and kidney.

A definite diagnosis of TSC includes clinical manifestations (Table III) (7) and genetic testing. The identification of a mutation (in either TSC1 or TSC2) is sufficient to diagnose TSC, without clinical manifestation. A pathogenic mutation is defined as a mutation that inactivates the function of the TSC1 or TSC2 proteins (out-of-frame indel or non-sense mutation), prevents protein synthesis (large genomic deletion),. Other TSC1 or TSC2 variants which exhibit a less certain effect on protein function do not meet these criteria and are not sufficient to make a definite diagnosis of TSC. Between 10 and 

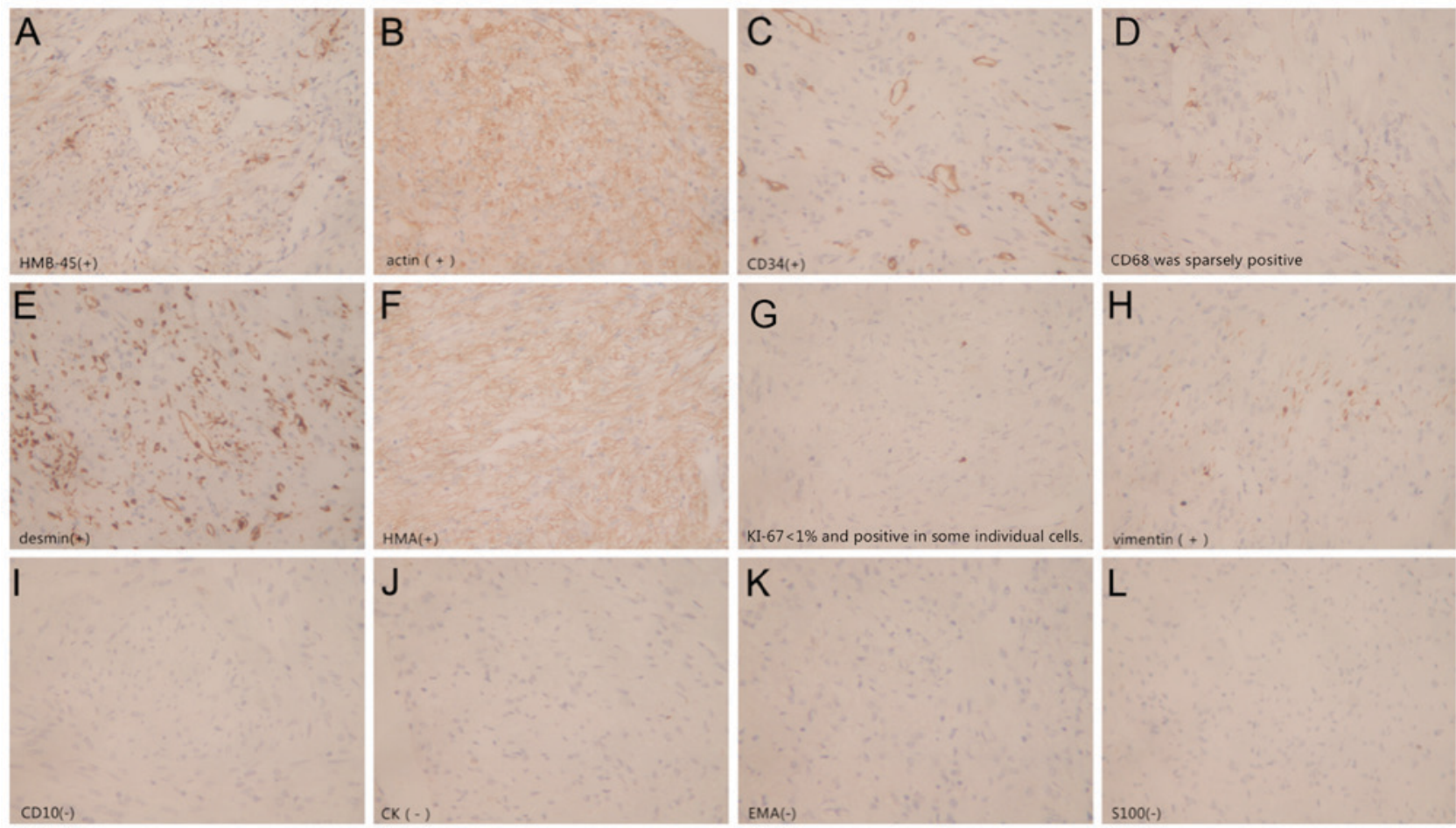

Figure 4. Immunochemistry of patient 3. (A) HMB-45(+); (B) actin(+); (C) CD34(+); (D) CD68 was sparsely positive; (E) desmin(+); (F) 5-(N, N-Hexamethylene) amiloride (HMA)(+); (G) Ki-67<1\% and positive in some individual cells; (H) vimentin(+); (I) CD10(-); (J) CK(-); (K) EMA(-); (L) S-100(-) (magnification, x10). HMB-45, homatropinemethylbromide 45; CD, cluster of differentiation; CK, cytokeratin; EMA, epithelial membrane antigen.
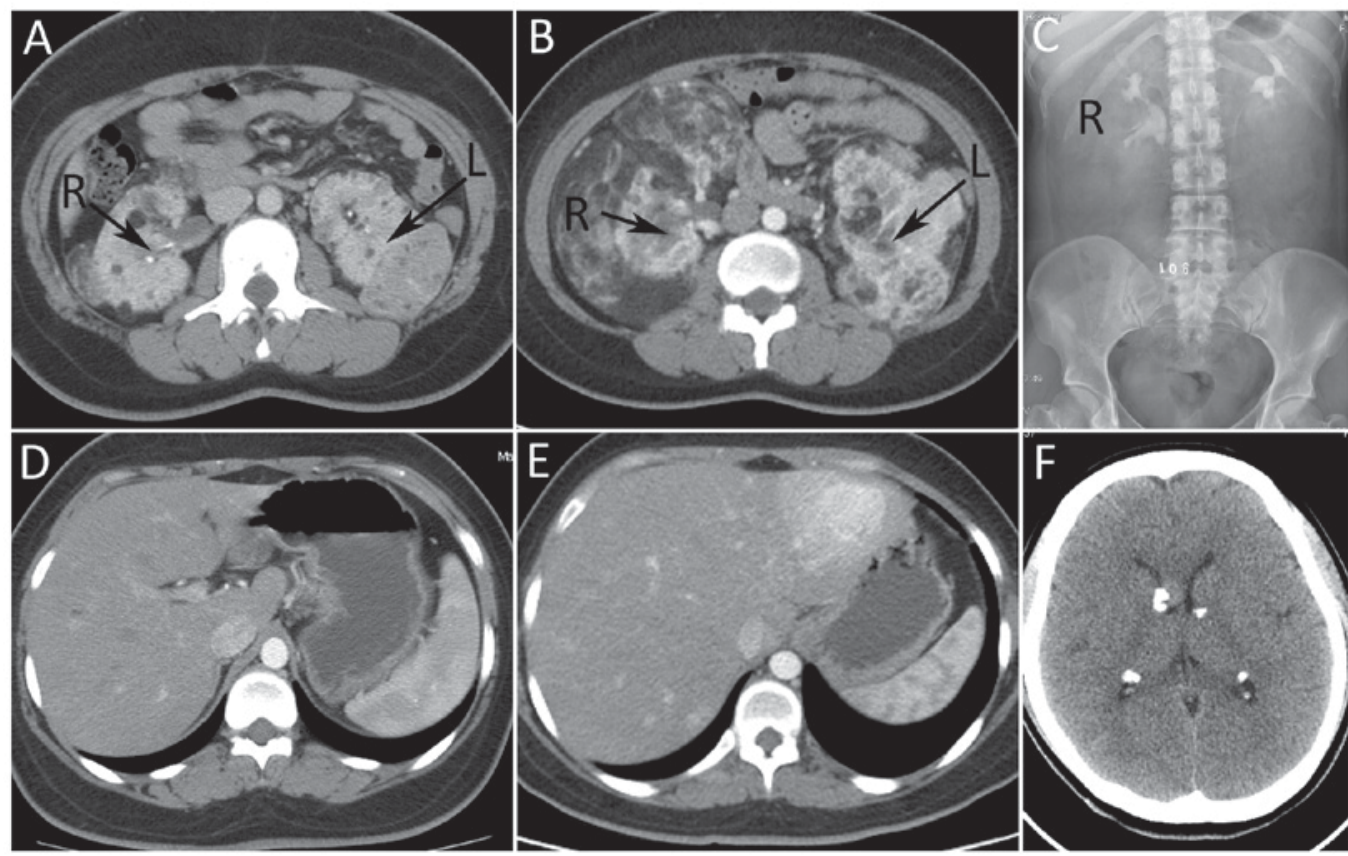

Figure 5. Imaging results of patient 4. (A) Renal CT 6 years prior (November 2009) revealed multiple AML in the two kidneys (diameter, 0.5-4 cm). (B) The number and volume of AML (6x5x4 cm on the left kidney, diffuse on the surface of the right kidney with a thickness of $\sim 4 \mathrm{~cm}$, May 2015), was increased significantly compared with that of 6 years ago $(4 \times 3 \times 3 \mathrm{~cm}$ on the left kidney, multiple tumors $<0.5 \mathrm{~cm}$ on the right kidney, November 2009). The left one is $15 \times 11 \times 8 \mathrm{~cm}$ and the right one is $17 \times 10 \times 9 \mathrm{~cm}$. CT value was between-80 and 23HU. (C) Intravenous urography revealed right upper calyceal and left inferior calyceal that were not developed. (D) An image from 6 years ago (November 2009) revealed no lesion in the liver of the patient prior; but (E) reveals multiple hemangioma developed 6 years following. (F) Head CT demonstrated subependymal giant cell astrocytoma in septum pellucidum near the interventricular foramen (diameter, $1.2 \mathrm{~cm}$ ) and multiple calcification lesions were identified. CT, computed tomography; AML, angiomyolipoma.

$25 \%$ of patients with TSC exhibit no mutation identified by conventional genetic testing; therefore, a normal genetic result does not exclude TSC (7).
Genetic testing maybe used for a definitive diagnosis of a patient with suspected TSC and for early diagnosis (compared with clinical manifestation), in particular for the early detection 
of the offspring of patients, which enables early intervention. The clinical manifestations often depend on the changes in the mutational gene. Although there have been a number of studies on TSC gene detection, this is not typically used for diagnosis in China due to its expensive costs. Furthermore, patients in the familial case could not afford genetic testing and did not wish to undergo it. The patients with TSC discussed in the present case report are from the same family, but there is no genetic diagnostic evidence for whether TSC is hereditary. It was hypothesized that all or some of the patients in this family developed TSC due to gene mutation.

The development of TSC RAML is slow, but due to the lack of specific treatment, the prognosis is unfavorable (13). Classical treatments include partial nephrectomy, selective renal arterial embolization, radiofrequency and cryotherapy (14). Previous studies on mTOR inhibitors, including everolimus and sirolimus, indicate that these effectively decreased tumor size of RAML, subependymal giant cell astrocytoma and lymphangioleiomyomatosis (15-17). It has been identified that using the mTOR inhibitor combined with the classical treatments may aid controlling the disease and decrease the adverse reactions of drugs $(14,16,18)$.

It has been demonstrated that the incidence rate of neurological disease associated with TSC are as follows: Epilepsy, between 66 and 93\%; subependymal nodules, between 90 and $100 \%$; subependymal giant cell astrocytoma, between 5 and 20\%; intelligent degradation, between 44 and 64\%; and infantile spasms, $45 \%$ (19). A previous study identified that $90 \%$ of cases of TSC-associated epilepsy were caused by brain tumors (20). Patients 1 and 3 had a history of epilepsy, but head CTs of the two patients revealed only partial calcification. The head CT of patient 4 suggested subependymal giant cell astrocytoma but there was no history of epilepsy; this phenomenon rarely happens and is difficult to explain, and the traditional anti-epilepsy therapies have limited effect. It has been revealed that mTOR inhibitors may decrease seizure frequency, improve learning deficits and reduce the size of tumor in order to relieve compression of tumor on its surrounding brain tissue (15).

The incidence of TSC has been identified to exhibit no significant association with the age or sex of patients (21) and the majority of patients develop clinical symptoms after 3 years of age (22). Clinical manifestations are delayed, whereas a genetic diagnosis may reveal TSC earlier. Therefore, clinical symptoms are of limited use in the early diagnosis of the disease. Patient 1 in the present study had suffered from epilepsy since the age of 2, which is rare. It has been demonstrated that RAML expresses receptors of estrogen and lutein, and tumors develop more rapidly in pregnant women (22-24). In addition, administration of mTOR inhibitors has led to female adolescent amenorrhoea (25-27). However, Sauter et al (28) considered that the use of drugs containing estrogenis not associated with renal angiomyolipoma in tuberous sclerosis. Additional research is required to understand if there is any association between the two.

A total of $80 \%$ of patients with TSC are complicated with RAML, with females exhibiting an increased incidence of TSC RAML compared with males (22); however, the severity of the illness remains unknown. AML is divided into classical and epithelioid AML. Patients with TSC may exhibit classical or epithelioid AML, but the incidence of epithelioid AML is increased compared with that of the classical type (29). Pathologically, epithelioid RAML exhibits histological variances with tumor cells demonstrating destructive and invasive development, and malignant tendency. Patient 3 exhibited classical AML with no proliferation of epithelioid cells, necrotized tumor tissue, nuclear division, atypia or invasive growth under microscopy. TSC-associated renal manifestations include cysts, angiomyolipoma, epithelioid tumor, renal cell carcinoma and eosinophilic cell tumor, and may coexist or exist independently (30-33). The incidence of TSC-associated RAML is $20 \%$ of RAML and typically develops bilaterally (34). Imaging techniques enable TSC RAML to be distinguished from the classical type. A tumor of the former type develops destructively in multiple centers and typically involves two sides, whereas the classical type develops separately and involves only one side. Patients with bilateral giant renal hamartoma are rare globally. To the best of our knowledge, the largest bilateral TSC RAML (right side) identified was $32 \times 16 \times 12 \mathrm{~cm}$ in size and had a mass of $7.7 \mathrm{~kg}$ (35). In addition, the largest unilateral renal AML (left side) identified was 39x25x9 cm in size with a mass of $7.5 \mathrm{~kg}$ (36). TSC RAML is typically accompanied with an aneurysm and with the developmentof the RAML, aneurysm increases. If a tumor is $>4 \mathrm{~cm}$, or the diameter of aneurysm is $>5 \mathrm{~mm}$, the patient exhibits an increased risk of tumor rupture hemorrhage, which is the primary cause of TSC RAML-associated mortality (37,38). For patients with RAML, close follow-ups maybe advised if the tumor is $<4 \mathrm{~cm}$ and there are no clinical symptoms. Patients with tumors $>4 \mathrm{~cm}$, aneurysm with diameter is $>5 \mathrm{~mm}$ or clinical manifestations (hemorrhage, fever, abdominal pain, hypertension and hematuria subsequent to RAML) are administered treatment. A previous study identified that tumors with a diameter $>10 \mathrm{~cm}$ were termed 'giant AMLs', which are easily ruptured causing hemorrhage, and require increased attention (39). For patients with TSC RAML that rupture to form hemorrhages, selective renal arterial embolization may be the selected treatment. Although there was no obvious hemorrhage in the giant RAML of the patient in this case, low hemoglobin suggested possible slow hemorrhage with small vessel rupture.

The patients in the present case report exhibited no renal function failure and a previous study revealed that renal failure rarely occurs in TSC (21). Thus, it was presumed that possibly residual normal renal tissue, which is difficult to distinguish with the naked eye, serves an important role in retaining renal function, but it is difficult to explain how a limited amount of tissue is able to retain normal renal function. It is hypothesized that genetic mutation causes normal renal tissue to be replaced by abnormal components, which exhibit physiological functions similar to normal renal tissue. However, further study is required.

In symptomatic TSC, clinical intervention is administered in the majority of cases to decrease the incidence rate of rupture hemorrhage and preserve renal function. A previous study identified that there are $>1,300$ types of TSC 1 and TSC 2 mutations (40), which may be associated with the different clinical manifestations between patients with TSC. The mutation rate of TSC2 in the patients with TSC is significantly increased and the associated clinical manifestations are of increased severity compared with that of TSC1 (33). The clinical application of 
mTOR inhibitors in the treatment of TSC has been revealed to achieve a therapeutic effect (41). mTOR inhibitors may be used to treat patients with TSC that exhibit subependymal giant cell astrocytoma, lymphangioleiomyomatosis, RAML and facial angiofiromasin due to the immunosuppressive actions exhibited on malignant cells (26). Previous studies have revealed that regular medication for 1 year significantly decreased the volume of RAML $(42,43)$. In larger tumors, the effect was more significant; however, renal tissue damaged by tumors may not always be recovered, in addition, there is a chance of tumor relapse following drug withdrawal (44) and the rate of AML rupture hemorrhage may not decrease.

The adverse reactions of mTOR inhibitors include oral cavity ulcers, stomatitis, convulsion, an acne-like rash, joint pain, fever, respiratory tract infection and skin lesions, and the severity of adverse reactions depends on the dose of the drug $(16,39)$. A previous study identified that the side-effects of drug treatment alone are strong, but the combination of classical treatment and a small dose of mTOR inhibitors may achieve improved results (27). In addition, in cases of RAML where surgery may be required, the administration of an mTOR inhibitor prior to surgery may decrease the tumor volume and the difficulty of surgery (16). Small dose maintenance therapy subsequent to surgery may exhibit improve the maintenance of the surgery result, control disease progression and decrease tumor recurrence $(16,45)$. Percutaneous cryoablation therapy is typically used in the treatment of renal cell carcinoma, but is rarely used in RAML. To the best of our knowledge, only one study has demonstrated that percutaneous cryoablation may be combined with a small dose of mTOR inhibitors to treat TSC RAML to achieve a curative effect and a decrease in adverse drug reactions (14).

The bilateral renal morphology of patient 4 was acceptable, the RAML progressed rapidly 6 years subsequently with the development of tumors in multiple centers and almost all the normal kidney morphology was absent. If mTOR inhibitors had been administered in the early stage, prior to the alterations of the kidney morphology, normal renal tissue may have been preserved, the development of a subependymal astrocytoma in the brain may have been prevented and the facial angiofibroma may have been treated. Therefore, mTOR inhibitors may have decreased the development of the disease and improved the quality of life for the patient. A previous study has indicated that early application of the drug treatment, particularly in infants and young children, may prevent tumor development, improve the symptoms of epilepsy and other TSC-associated symptoms (15). All patients in the present case report were not administered mTOR inhibitors due to the increased cost associated with this treatment, which health insurers in China do not cover. Therefore, the present case report lacks information on the clinical application of this class of drug, and there are a limited number of studies on the association between estrogen, mTOR inhibitors and disease.

mTOR inhibitors may be used in the treatment of TSC-associated diseases, but cannot prevent the disease being genetically passed on to the children of patients. Therefore, it is recommended that patients with TSC have a fetal gene detection performed during pregnancy. For the treatment of patients with TSC, early application of drug treatment is advised as this may prevent the development of tumors, epilepsy and other diseases associated with TSC. In addition, regular re-examination is important because the progression speed of TSC is distinct, depending on the individual patient. Tumors in certain patients with TSC have been revealed to develop dominantly in a short term, but there have additionally been patients with TSC that experience long-term survival with a tumor (15). Patients with TSC with rapidly developing tumors may be administered mTOR inhibitor therapy combined with surgery, arterial embolization, radiofrequency ablation or cryoablation. Novel combination treatment methods include small dose mTOR inhibitor with partial renal nephrectomy and small dose of mTOR inhibitor maintenance treatment, or arterial embolization with partial renal nephrectomy and mTOR inhibitor, or mTOR inhibitor with cyroablation, all of which may exhibit therapeutic prospects in the future. Additional research is required. The present case report described rare cases in order to provide more appropriate diagnostic and therapeutic methods for patients with TSC. These include fetal gene detection during early pregnancy, early application of drugs and the combination of multiple treatments.

\section{References}

1. Zhang Y, Gan J, Pu Z, Xu Mm, Wang Lf, Li Yh and Liu Zg: TSC1 R509X Mutation in a chinese family with tuberous sclerosis complex. Neuromolecular Med 17: 202-208, 2015.

2. Cabrera-López C, Bullich G, Marti T, Català V, Ballarín J, Bissler JJ, Harris PC, Ars E and Torra R: Insight into response to mTOR inhibition when PKD1 and TSC2 are mutated. BMC Med Genet 16: 39, 2015.

3. Hallett L, Foster T, Liu Z, Blieden M and Valentim J: Burden of disease and unmet needs in tuberous sclerosis complex with neurological manifestations: Systematic review. Curr Med Res Opin 27: 1571-1583, 2011.

4. Hyman MH and Whittemore VH: National Institutes of Health consensus conference: Tuberous sclerosis complex. Arch Neurol 57: 662-665, 2000.

5. Rabito MJ and Kaye AD: Tuberous sclerosis complex: Perioperative considerations. Ochsner J 14: 229-239, 2014.

6. Wang GX, Wang DW, Yi CY, Qu JS and Wang YL: Mutational analyses of the TSC1 and TSC 2 genes in cases of tuberous sclerosis complex in Chinese Han children. Genet Mol Res 12: 1168-1175, 2013.

7. Northrup $\mathrm{H}$ and Krueger DA; International Tuberous Sclerosis Complex Consensus Group: Tuberous sclerosis complex diagnostic criteria update: Recommendations of the 2012 iinternational tuberous sclerosis complex consensus conference. Pediatr Neurol 49: 243-254, 2013.

8. Sancak O, Nellist M, Goedbloed M, Elfferich P, Wouters C, Maat-Kievit A, Zonnenberg B, Verhoef S, Halley D and van den Ouweland A: Mutational analysis of the TSC1 and TSC2 genes in a diagnostic setting: Genotype-phenotype correlations and comparison of diagnostic DNA techniques in tuberous sclerosis complex. Eur J Hum Genet 13: 731-741, 2005.

9. van Slegtenhorst M, de Hoogt R, Hermans C, Nellist M, Janssen B, Verhoef S, Lindhout D, van den Ouweland A, Halley D, Young J, et al: Identification of the tuberous sclerosis gene TSC1 on chromosome 9q34. Science 277: 805-808, 1997.

10. Habib SL: Tuberous sclerosis complex and DNA repair. Adv Exp Med Biol 685: 84-94, 2010.

11. Inoki K, Corradetti MN and Guan K L: Dysregulation of the TSC-mTOR pathway in human disease. Nat Genet 37: 19-24, 2005.

12. Henske EP, Rasooly R, Siroky B and Bissler J: Tuberous sclerosis complex, mTOR, and the kidney: Report of an NIDDK-sponsored workshop. Am J Physiol Renal Physiol 306: F279-F283, 2014.

13. Yi L, Guo F and Zhang YB: Diagnosis and analysis of cases of tuberous sclerosis. J Clin Exp Med 10: 819-822, 2015 (In Chinese).

14. Krummel T, Garnon J, Lang H, Gangi A and Hannedouche T: Percutaneous cryoablation for tuberous sclerosis-associated renal angiomyolipoma with neoadjuvant mTOR inhibition. BMC Urol 14: 77, 2014. 
15. Curatolo $P$ and Moavero R: mTOR inhibitors in tuberous sclerosis complex. Curr Neuropharmacol 10: 404-415, 2012.

16. Staehler M, Sauter M, Helck A, Linsenmaier U, Weber L, Mayer K and Fischereder M: Nephron-sparing resection of angiomyolipoma after sirolimus pretreatment in patients with tuberous sclerosis. Int Urol Nephrol 44: 1657-1661, 2012.

17. Sasongko TH, Ismail NF and Zabidi-Hussin Z: Rapamycin and rapalogs for tuberous sclerosis complex. Cochrane Database Syst Rev 7: CD011272, 2016.

18. Peces R, Cuestalópez E, Peces C and Selgas R: Giant bilateral renal angiomyolipomas and lymphangioleiomyomatosis presenting after two successive pregnancies successfully treated with surgery and rapamycin. ScientificWorldJournal 11: 2115-2123, 2011.

19. Hallett L, Foster T, Liu Z, Blieden M and Valentim J: Burden of disease and unmet needs in tuberous sclerosis complex with neurological manifestations: Systematic review. Curr Med Res Opin 27: 1571-1583, 2011.

20. Crino PB, Nathanson KL and Henske EP: The tuberous sclerosis complex. N Engl J Med 355: 1345-1356, 2006.

21. O'Callaghan FJ, Noakes MJ, Martyn CN and Osborne JP: An epidemiological study of renal pathology in tuberous sclerosis complex. BJU Int 94: 853-857, 2004.

22. Mascarenhas R and McLaughlin P: Haemorrhage from angiomyolipoma of kidney during pregnancy-a diagnostic dilemma. Ir Med J 94: 83-84, 2001.

23. Raft J, Lalot JM, Meistelman C and Longrois D: Influence of pregnancy on renal angiomyolipoma. Gynecol Obstet Fertil 33: 898-906, 2005 (In French).

24. Boorjian SA, Sheinin Y, Crispen PL, Lohse CM, Kwon ED and Leibovich BC: Hormone receptor expression in renal angiomyolipoma: Clinicopathologic correlation. Urology 72: 927-932, 2008

25. Bissler JJ, Kingswood JC, Radzikowska E, Zonnenberg BA, Frost M, Belousova E, Sauter M, Nonomura N, Brakemeier S, de Vries PJ, et al: Everolimus for angiomyolipoma associated with tuberous sclerosis complex or sporadic lymphangioleiomyomatosis (EXIST-2): A multicentre, randomised, double-blind, placebo-controlled trial. Lancet 381: 817-824, 2013.

26. Franz DN: Everolimus in the treatment of subependymal giant cell astrocytomas, angiomyolipomas, and pulmonary and skin lesions associated with tuberous sclerosis complex. Biologics 7: 211-221, 2013.

27. Roa J, Garcia-Galiano D, Castellano JM, Gaytan F, Pinilla L and Tena-Sempere M: Metabolic control of puberty onset: New players, new mechanisms. Mol Cell Endocrinol 324: 87-94, 2010

28. Sauter M, Sigl J, Schotten KJ, Kreuzer L, Günthner-Biller M and Fischereder M: Use of oestrogen-containing medication is not associated with renal angiomyolipoma in tuberous sclerosis: Findings from a survey. Int Urol Nephrol 47: 707-708, 2015.

29. Huang KH, Huang CY, Chung SD, Pu YS, Shun CT and Chen J: Malignant epithelioid angiomyolipoma of the kidney. J Formos Med Assoc 106 (2 Suppl): S51-S54, 2007.

30. Kavaney PB and Fielding I: Angiomyolipoma and renal cell carcinoma in same kidney. Urology 6: 643-646, 1975.
31. Corsenca A, Aebersold F, Moch H, Bird P, Weber M, Hofbauer G, Wüthrich RP and Serra AL: Combined nephrectomy and pre-emptive renal transplantation in a tuberous sclerosis patient with angiomyolipoma, renal carcinoma and life-threatening abdominal haemorrhages. Nephrol Dial Transplant 22: 3330-3333, 2007.

32. Eisenhauer EA, Therasse P, Bogaerts J, Schwartz LH, Sargent D, Ford R, Dancey J, Arbuck S, Gwyther S, Mooney M, et al: New response evaluation criteria in solid tumours: Revised RECIST guideline (version 1.1). Eur J Cancer 45: 228-247, 2009.

33. Eble JN: Angiomyolipoma of kidney. Semin Diagn Pathol 15: 21-40, 1998.

34. Cichocki M, Sosnowski M and Jablonowski Z: A giant renal angiomyolipoma (AML) in a patient with septo-optic dysplasia (SOD). Eur J Med Res19: 46, 2014.

35. Hussain M, Mubarak M, Sultan G, Sultan G, Ahmed E, Yunus M, Salehi MA, Asif M, Anwer Naqvi SA and Rizvi SA: Renal transplant in a tuberous sclerosis patient with bilateral giant renal angiomyolipomas and concurrent renal carcinoma. Saudi J Kidney Dis Transpl 24: 318-321, 2013

36. Taneja R and Singh DV: Giant renal angiomyolipoma: Unusual cause of huge abdominal mass. J Clin Imaging Sci 3: 56, 2013.

37. Miyoshi Y, Iwao K, Nawa G, Yoshikawa H, Ochi T and Nakamura Y: Frequent mutations in the beta-catenin gene in desmoid tumors from patients without familial adenomatous polyposis. Oncol Res 10: 591-594, 1998.

38. Yamakado K, Tanaka N, Nakagawa T, Kobayashi S, Yanagawa M and Takeda K: Renal angiomyolipoma: Relationships between tumor size, aneurysm formation, and rupture. Radiology 225: 78-82, 2002.

39. Mitra A, Kumar Jain S, Gupta D and Chandra Murthy Kaza R: Giant angiomyolipoma of the kidney presenting as anaemia-a rare presentation. Open J Urol 2: 75-77, 2012.

40. Curatolo P, Bombardieri R and Jozwiak S: Tuberous sclerosis. Lancet 372: 657-668, 2008.

41. Yang G, Yang L, Yang X, Shi X, Wang J, Liu Y, Ju J and Zou L: Efficacy and safety of a mammalian target of rapamycin inhibitor in pediatric patients with tuberous sclerosis complex: A systematic review and meta-analysis. Exp Ther Med 9: 626-630, 2015.

42. Bissler JJ, McCormack FX, Young LR, Elwing JM, Chuck G, Leonard JM, Schmithorst VJ, Laor T, Brody AS, Bean J, et al: Sirolimus for angiomyolipoma in tuberous sclerosis complex or lymphangioleiomyomatosis. N Engl J Med 358: 140-151, 2008.

43. Cabrera-López C, Martí T, Catalá V, Torres F, Mateu S, Ballarín J and Torra R: Assessing the effectiveness of rapamycin on angiomyolipoma in tuberous sclerosis: A two years trial. Orphanet J Rare Dis 7: 87, 2012.

44. Peng ZF, Yang L, Wang TT, Han P, Liu ZH and Wei Q: Efficacy and safety of sirolimus for renal angiomyolipoma in patients with tuberous sclerosis complex or sporadic lymphangioleiomyomatosis: A systematic review. J Urol 192: 1424-1430, 2014.

45. Paul E and Thiele E: Efficacy of sirolimus in treating tuberous sclerosis and lymphangioleiomyomatosis. N Engl J Med 358: 190-192, 2008 